\title{
Tailored therapy in patients treated with fluoropyrimidines: focus on the role of dihydropyrimidine dehydrogenase
}

\author{
Filippo Merloni ${ }^{1}$, Nicoletta Ranallo ${ }^{1}$, Laura Scortichini ${ }^{1}$, Riccardo Giampieri ${ }^{2}$, Rossana Berardi ${ }^{2}$ \\ 'Scuola di Specializzazione in Oncologia, Università Politecnica delle Marche, Ancona 60121, Italy. \\ ${ }^{2}$ Clinica Oncologica, Università Politecnica delle Marche, AOU Ospedali Riuniti, Ancona 60126, Italy.
}

Correspondence to: Prof. Berardi Rossana, Clinica Oncologica, Università Politecnica delle Marche, AOU Ospedali Riuniti, Via Conca 71, Ancona 60126, Italy. E-mail: rossana.berardi.it@gmail.com; r.berardi@univpm.it

How to cite this article: Merloni F, Ranallo N, Scortichini L, Giampieri R, Berardi R. Tailored therapy in patients treated with fluoropyrimidines: focus on the role of dihydropyrimidine dehydrogenase. Cancer Drug Resist 2019;2:787-802. http://dx.doi.org/10.20517/cdr.2018.006

Received: 15 Dec 2018 First Decision: 14 Feb 2019 Revised: 1 Mar 2019 Accepted: 4 Jun 2019 Published: 19 Sep 2019

Science Editor: Godefridus J. Peters Copy Editor: Huan-Liang Wu Production Editor: Jing Yu

\begin{abstract}
Fluoropyrimidines are widely used in the treatment of solid tumors, mainly gastrointestinal, head and neck and breast cancer. Dihydropyrimidine dehydrogenase (DPD) is the rate-limiting enzyme for catabolism of 5-FU and it is encoded by DPYD gene. To date, many known polymorphisms cause DPD deficiency and subsequent increase of 5-FU toxicity. In addition, reduced inactivation of 5-FU could lead to increased 5-FU intracellular concentration and augmented efficacy of this drugs. Therefore DPD expression, particularly intratumoral, has been investigated as predictive and prognostic marker in 5-FU treated patients. There also seems to be a tendency to support the correlation between DPD expression and response/survival in patients treated with fluoropyrimidine even if definitive conclusions cannot be drawn considering that some studies are conflicting. Therefore, the debate on intratumoral DPD expression as a potential predictor and prognostic marker in patients treated with fluoropyrimidines is still open. Four DPD-polymorphisms are the most relevant for their frequency in population and clinical relevance. Many studies demonstrate that treating a carrier of one of these polymorphisms with a full dose of fluoropyrimidine can expose patient to a severe, even life-threatening, toxicity. Severe toxicity is reduced if this kind of patients received a dose-adjustment after being genotyped. CPIC (Clinical Pharmacogenetics Implementation Consortium) is an International Consortium creating guidelines for facilitating use of pharmacogenetic tests for patient care and helps clinicians ensuring a safer drug delivery to the patient. Using predictive DPD deficiency tests in patients receiving 5FU-based chemotherapy, in particular for colorectal cancer, has proven to be a cost-effective strategy.
\end{abstract}

\footnotetext{
(@) (-)

(C) The Author(s) 2019. Open Access This article is licensed under a Creative Commons Attribution 4.0 International License (https://creativecommons.org/licenses/by/4.0/), which permits unrestricted use, sharing, adaptation, distribution and reproduction in any medium or format, for any purpose, even commercially, as long as you give appropriate credit to the original author(s) and the source, provide a link to the Creative Commons license, and indicate if changes were made.
}

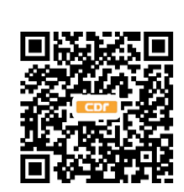


Keywords: 5-fluorouracil, fluoropyrimidines, chemotherapy, dihydropyrimidine dehydrogenase, dihydropyrimidine dehydrogenase, $D P Y D, C P I C$, polymorphisms

\section{INTRODUCTION}

Fluoropyrimidines (FU) are the most prescribed anticancer drugs for the treatment of solid cancers, in particular breast, colorectal, head and neck, pancreas and gastric cancers ${ }^{[1,2]}$. The most common side effects are represented by emesis, bone marrow suppression, diarrhea, mucositis, fatigue and hand-foot syndrome. Fluoropyrimidines cause severe toxicities in $10 \%$ to $40 \%$ of patients and deaths in $0.2 \%$ to $0.8 \%{ }^{[3]}$.

The correct management of fluoropyrimidine toxicity consists in the temporary suspension or interruption of treatment ${ }^{[4-7]}$. The metabolic pathway of 5 -fluorouracil depends on the activity of many intracellular enzymes including dihydropyrimidine dehydrogenase (DPD) $)^{[1]}$.

DPD expression varies throughout several tissues and exerts his activity predominantly in liver, peripheral blood mononuclear cells, tumor and inflammatory tissues. Genotype can explain high evident interindividual variability of DPD levels and a circadian rhythm of expression of this enzyme is described $^{[8,9]}$.

On top of that, differences in DPD activity have been reported among different ethnic groups ${ }^{[10-12]}$. Patients who receive 5-FU-based chemotherapy usually eliminate over $80 \%$ of this drug by converting it into its inactive metabolite 5,6-dihydro-5-fluorouracil ${ }^{[4,13,14]}$, owing to levels of DPD within the normal range. In patients showing DPD deficiency, decreased catabolism of 5-FU is observed and thus, increased risk of toxicity ${ }^{[15]}$.

In addition to a major risk of toxicity, reduced inactivation of 5-FU, could theoretically lead to increased 5-FU intracellular concentration and augmented efficacy of this chemotherapeutic agent. Therefore DPD expression, especially intratumoral, assessed by either enzymatic activity or mRNA expression, has been investigated as a predictive and prognostic marker in 5-FU treated patients, especially in those affected by colorectal cancer (CRC) given the prominent role of this chemotherapeutic agents in both metastatic and adjuvant setting.

In this review we will discuss the impact of 5-FU pharmacogenomics in the clinical outcome of patients treated with fluorouracil-based chemotherapy and in particular we will analyze the importance of polymorphisms of the enzyme DPD regarding the toxicity and efficacy of chemotherapy 5FU-based. We also want to underline how the prospective analysis of DPD mutations has a good cost-effectiveness ratio.

\section{FLUOROURACIL METABOLISM AND DPD}

\section{Fluoropyrimidines include 5-FU and its oral products capecitabine and tegafur}

5-FU is a cytotoxic agent that belongs to the class of antimetabolites. It is administered intravenously and has a short half-life (10-15 min). 5-FU undergoes a series of bio-transformative reactions that convert it into nucleotide metabolites; one of these metabolites forms a complex with the enzyme thymidylate synthase with inhibition of DNA synthesis through a "thymineless death" mechanism. Capecitabine and tegafur are pro-drugs of 5-FU; they have a bioavailability of approximately $70 \%-80 \%$ after oral $\operatorname{administration}^{[2,3,14]}$.

Only a small fraction of 5-FU (1\%-5\%) is converted into cytotoxic metabolites. More than $80 \%$ of the $5-\mathrm{FU}$ undergoes hepatic metabolism by the DPD enzyme whose action consists in the transformation of 5-FU 
into its inactive product dihydrofluorouracil which is degraded and eliminated through the urine. This causes the DPD to be the rate-limiting step of the inactivation of 5-FU. Other factors such as age, race, comorbidities and concomitant therapies can influence metabolism ${ }^{[5,13,14]}$.

The activity of the DPD presents numerous inter-individual variations. In about 3\%-8\% of the population there is a partial lack of DPD expression which determines a reduction of the enzymatic activity of about $50 \%{ }^{[15,16]}$. Approximately $0.1 \%$ has a complete DPD deficit; this implies null enzymatic activity ${ }^{[10,16-18]}$.

In patients that receive 5-FU-based therapy and who have partial DPD deficiency, decreased enzymatic activity results in a reduction in 5-FU inactivation and also in a high risk of severe or fatal toxicity ${ }^{[15,19]}$. Retrospective assessment of patients who developed severe toxicity during treatment with 5-FU, showed that $39 \%-61 \%$ had reduced activity of DPD enzyme ${ }^{[20-23]}$. The DPD is encoded by the DPYD gene located on chromosome 1 p22 and composed of 23 exons $^{[24]}$. DPD deficiency is usually the result of genetic polymorphisms of DPYD. DPYD is a highly polymorphic gene; over 50 polymorphic variants have been described $^{[20,25]}$. The screening of the DPYD variants is a valuable aid in clinical practice in predicting the occurrence of any toxicity in patients with DPD deficiency ${ }^{[25,26]}$.

\section{DPD AND TOXICITY}

It is confirmed that genetic variation of DPYD, that leads to DPD deficiency, can cause severe toxicity in patients treated with a fluoropyrimidine-based chemotherapy. Because of the high doses administered, DPD-deficient patients are likely to experience life-threatening toxicities ${ }^{[27]}$. This pharmacogenetic "DPD syndrome" manifests typically as severe or fatal diarrhea, mucositis/stomatitis, myelosuppression and even rarer toxicities, such as hepatitis and encephalopathy ${ }^{[28]}$. It is hypothesized that safety could be improved by prospective evaluation of DPD-deficiency before treatment start and through adequate dose-adjustment.

Many different methods are used to test DPD deficiency and they can be mainly divided into three groups: tests aimed at assessing DPD enzyme activity, mRNA variants and genetic variants in DPYD gene ${ }^{[29]}$.

In the routine the best way to identify DPD-deficient patients is measurement of physiological plasma dihydrouracil/uracil ( $\left.\mathrm{UH}_{2} / \mathrm{U}\right)$ ratio. Analysis of uracil (U) and dihydrouracil (UH2) is performed in frozen plasma using a high-performance liquid chromatography with ultraviolet detection method; DPD deficiency is reflected by plasma $\mathrm{UH}_{2} / \mathrm{U}$ decrease or plasma uracil increase. Launay et al. ${ }^{[27,30]}$ demonstrated how upfront DPD testing with this ratio and tailored dosing can reduce the incidence of toxicities, while maintaining optimal efficacy in patients treated with 5-FU in a variety of settings.

Due to the difference in terms of enzymatic activity of each variant they will be treated separately in the following section.

\section{Dose individualization based on specific genotype}

Numerous genetic variants in DPYD gene are known to change the protein sequence or mRNA splicing process; the consequence is that some of them do not affect DPD activity, instead of other that can cause severe deficiency and related adverse events. Four variants are of primary relevance, because of their frequency in population and their clinical impact $\left(D P Y D^{\star} 2 \mathrm{~A}, D P Y D{ }^{\star} 13, \mathrm{c} .2846 \mathrm{~A}>\mathrm{T}\right.$ e c. $1129-5923 \mathrm{C}>\mathrm{G}$ $(\mathrm{HapB} 3)^{[31]}$.

$D P Y D^{*} 2$ A genotype (IVS14+1G>A; c.1905+1G>A; rs3918290) is the first described as being functionally relevant and the most studied polymorphism ${ }^{[32]}$. This allele frequency varies between $0.1 \%$ and $1 \%$ in African-American and Caucasian population respectively ${ }^{[33-36]}$. Its located at the intron of exon 14 and results in skipping of all the exon and in a nonfunctional protein ${ }^{[32,37]}$. 
"In vitro" studies showed several DPYD variants that were homozygous in mammalian cells; the enzymatic activity of DPD protein was absent when it was assessed in $D P Y D^{\star} 2 \mathrm{~A}$ carriers ${ }^{[38]}$. So, it can be assumed that heterozygous carriers of this variant have approximately half of enzyme activity.

Deenen et al. ${ }^{[26]}$ conducted a prospective clinical trial focused on DPYD*2A-guided dosing of fluoropyrimidines, as to prevent severe toxicity. Patients who would be treated with fluoropyrimidinebased chemotherapy were genotyped for $D P Y D^{\star} 2 \mathrm{~A}$ before treatment start: $D P Y D^{\star} 2 \mathrm{~A}$ variant allele carriers were treated with a starting dose reduction of $50 \%$. Then, if well tolerated, the patients would receive a dose-titration phase. Toxicity data for variant allele patients treated with a reduced dose were compared with controls from literature, i.e., $D P Y D^{\star} 2 \mathrm{~A}$ variant allele carriers receiving standard dose, since of the lack of a proper treatment arm with full-dose fluoropyrimidine-based chemotherapy: in particular, due to the already well-known association between $D P Y D^{\star} 2 \mathrm{~A}$ and increased risk of severe and lethal toxicity it was considered unethical to have such a treatment arm.

The study showed that risk of severe (grade $\geq 3$ ) treatment-related toxicity was significantly lower in $D P Y D^{\star} 2 \mathrm{~A}$ variant allele carriers undergoing genotype-guided dosing than in the historical controls, respectively, $28 \%$ and $73 \%(P<0.001)$. Drug-related death was reduced from $10 \%$ in historical controls to $0 \%$ in this study.

\section{Other three variants}

c.2846A>T variant allele (D949V; rs67376798) causes a structural change in DPD enzyme that leads to a deficient function ${ }^{[39]}$. Allele frequency varies from $0.1 \%$ in African-Americans to $1.1 \%$ in Caucasians ${ }^{[32,39-41]}$. In vitro data by Offer et al. ${ }^{[41]}$ reveal that patients with homozygous expression of this variant have a $59 \%$ activity, compared to WT. These results show that even if enzyme activity is significantly reduced it is also more active if compared to homozygous carriers of $D P Y D^{*} 2 \mathrm{~A}^{[37]}$.

We can deduce that if homozygous expression of c.2846A>T variant allele causes approximately $50 \%$ enzyme activity reduction, heterozygous carriers are likely to have $25 \%$ reduction in DPD activity; it is then suggested that they would advantage from a $25 \%$ drug dose reduction ${ }^{[34]}$.

c. $1679 \mathrm{~T}>\mathrm{G}\left(D P Y D^{*} 13\right.$; I560S; rs55886062) is a rare DPYD polymorphism: its frequency varies from $0.07 \%$ to $0.1 \%$ in Caucasians ${ }^{[32,40]}$. In vitro study by Offer et al ${ }^{[37]}$ demonstrated that homozygous carriers have a reduction of $75 \%$ in DPD enzyme activity and suggests that the heterozygous carriers would have around $50 \%$ reduction in DPD enzyme activity.

c.1236G $>$ A polymorphism (E412E; rs556038477) is in exon 11 and it is in complete linkage with all the variants named haplotype B3 (HapB3) (c.483+18G >A, c.680+139G $>A, c .959-51 \mathrm{~T}>\mathrm{G}$ and c.1129-5923C $>\mathrm{G})^{[42,43]}$. The c1129-5923C $>\mathrm{G}$ intronic polymorphism (rs75017182) located deep in intron 10 causes abnormal splicing and maybe it is responsible for the DPD enzyme deficiency ${ }^{[31,43]}$.

The frequency of heterozygous patients in Caucasian populations varies between $2.6 \%$ and $6.3 \%{ }^{[42-46]}$. DPD activity is not completely absent in homozygous carriers; so it is expected that a $25 \%$ dose reduction for heterozygous carriers is convenient ${ }^{[34,4]}$.

The clinical validity of all these three variants, in particular the toxicity related to fluoropyrimidine-based chemotherapy in patients carriers of these mutations, was studied by Meulendijks et al. ${ }^{[48]}$ in a systematic review and meta-analysis. They collected data from 7356 patients and demonstrated that c.1679T $>\mathrm{G}$, c. $1236 \mathrm{G}>\mathrm{A}$, and c.2846 $\mathrm{A}>\mathrm{T}$ are clinically relevant predictors of fluoropyrimidine-associated toxicity, as the $D P Y D^{\star} 2 \mathrm{~A}$ variant. 
Table 1. Dose-reduction based on DPYD variant

\begin{tabular}{lc}
\hline DPYD variant & \% of standard fluoropyrimidine dose \\
\hline$D P Y D * 2 A(r s 3918290)$ & $50 \%$ \\
$c .1679 T>G(r s 55886062)$ & $50 \%$ \\
c. $2846 A>T(r s 67376798)$ & $75 \%$ \\
$c .1236 \mathrm{G}>\mathrm{A} / \mathrm{HapB3}(\mathrm{rs} 56038477)$ & $75 \%$ \\
\hline
\end{tabular}

On the base of these findings, it can be expected that initial dose reductions in heterozygous carriers of these three other DPYD polymorphisms will result in reduction of toxicity ${ }^{[49]}$ [Table 1].

Henricks et al. ${ }^{[50]}$ published a multicenter prospective study to investigate the effect of doseindividualization chemotherapy, on the basis of four DPYD variants, on fluoropyrimidine drugs toxicity. Patients were genotyped for the four variants of DPYD and received an initial dose reduction of 5-FU following the current guidelines ${ }^{[31]}$. This study shows that dose-individualization is achievable and can improve patient safety: it reduces risk of severe toxicity for $D P Y D^{\star} 2 \mathrm{~A}$ carriers, it is safe in c.1679T>G variant and decrease the toxicity risk in c.2846A $>\mathrm{T}$, although the risk was still higher than WT. For c. $1236 \mathrm{G}>\mathrm{A}$ carriers, a reduction of $25 \%$ is not enough to diminishing toxicity. When compared with patients in historical cohort, the frequency of severe toxicity in $D P Y D^{*} 2 \mathrm{~A}$ carriers was lower ( $31 \% v s$. $72 \%$ ); the only patient with c.1679 T $>$ G did not have toxicity after $50 \%$ dose reduction (the authors could not make correlation because of it was only one). For c.1236G>A and c. $2846 \mathrm{~A}>\mathrm{T}$ reduction of $25 \%$ seemed not safe: $39 \%$ of the first group and $47 \%$ of the second had severe toxicity. The authors proved even that dose adjustment chemotherapy can reduces hospital admission due to severe toxicity and therapy discontinuation of treatment. The authors compared the patients treated with genotyped-dose reduction $\mathrm{RR}$ for severe toxicity with the same RR in DPYD variant allele carriers from a historical cohort from a meta-analysis, (in this last study, patients were not genotyped before treatment and received a full dose chemotherapy $)^{[48]}$.

\section{IMPACT OF DPD EXPRESSION IN DIFFERENT CANCER TYPES}

\section{Colorectal cancer}

The role of DPD expression as a predictor of effectiveness of 5-FU has been highly investigated in CRC, in comparison to other types of cancer. However, definitive consensus on its reliability as predictive factor has not been reached yet. Below the studies in the metastatic and adjuvant setting are disussed separately given the difference between advanced and localized disease in terms of prognosis and biology.

Some studies analyzed the level of response of advanced CRC to 5-FU chemotherapy in relation to DPD values. Vallböhmer et al ${ }^{[51]}$ demonstrated a statistically significant correlation between DPD expression and response to 5-FU based chemotherapy. This study enrolled 37 patients with metastatic CRC treated with first-line capecitabine whose intratumoral DPD mRNA levels were assessed using laser capture microdissection and real-time (RT) PCR. Patients that had at least 50\% tumor shrinkage at the CT scan were classified as responders to therapy. The results highlighted a significant association between high levels of intratumoral DPD and resistance to capecitabine while patients with lower mRNA expression levels of DPD showed better progression free survival (PFS).

A correlation between intratumoral gene expression levels of DPD and response to 5-FU was also reported by a study conducted by Salonga et al.$^{[52]}$. By using a pre-established cut-off value (DPD: b-actin, $2.5 \times 10^{3}$ ), authors confirmed the negative predictive value of high levels of DPD (patients who have higher levels of DPD show actually no response after 5-FU based therapy compared with patients with lower levels of DPD where a response rate of $50 \%$ was seen). 
In addition to studies that suggested a potential correlation between DPD expression and 5-FU-based chemotherapy activity, there are a few reports where this assumption was not proven, either in patients with metastatic or unresectable locally advanced colorectal cancer ${ }^{[53,54]}$.

Other studies have assessed the role of DPD expression values as a prognostic factor in metastatic CRC. A large Phase III study conducted by Koopman et al. ${ }^{[5]}$ (CAIRO) randomized 803 advanced CRC patients between sequential treatment (first-line: capecitabine, second-line: irinotecan, third-line: capecitabine plus oxaliplatin) or combination treatment (first-line capecitabine plus irinotecan, second-line: capecitabine plus oxliplatin). Retrospective evaluation of DPD expression by immunohistochemistry (IHC) was performed in formalin-fixed paraffin-embedded samples obtained from 556 patients. A statistically significant positive predictive value for low versus high DPD values was noted in 283 patients treated with capecitabine plus irinotecan, in terms of improved median PFS and overall survival (OS) ${ }^{[55]}$.

Yanagisawa et al. ${ }^{[53]}$ also highlighted a positive correlation of low DPD expression and OS in a small number of patients affected metastatic CRC treated with a regimen composed by 5 -FU, irinotecan and leucovorin (MIFL). Further studies evidenced a benefit in terms of $\mathrm{PFS}^{[51]}$ and time to progression ${ }^{[56]}$ in patients with low DPD expression treated respectively with capecitabine and first-line 5-FU based regimen.

The potential influence of the DPD expression on outcome in patients treated with 5-FU based chemotherapy has been also investigated in adjuvant setting.

Ciaparrone et al. ${ }^{[57]}$ investigated the prognostic role of DPD values (evaluated by IHC) in 62 patients who underwent surgery and received 5-FU adjuvant treatment. At multivariate analysis, high DPD expression was significantly correlated with worse DFS and OS. Further studies reported a shorter DFS ${ }^{[58-60]}$ and both worse DFS and $\mathrm{OS}^{[61]}$ in patients with high DPD expression. Some studies evidenced that low DPD expression levels were associated with trends for better $\operatorname{OS}^{[0,66]}$ even if not statistically significant. Kornmann et al. ${ }^{[63]}$ examined the prognostic value of thymidylate synthase (TS) and DPD expression in 295 patients who received adjuvant 5-FU based chemotherapy after surgery. Patients who had tumors with low TS and high DPD levels had worse prognosis in contrast with the ones who expressed high TS and low DPD.

Nevertheless, some other studies, reported no correlation between DPD expression and prognosis in this setting ${ }^{[6-66]}$. These results taken together seem to suggest a correlation between low DPD expression and better prognosis in patients with colorectal cancer treated with 5-FU based chemotherapy in both metastatic [Table 2] and adjuvant setting [Table 3]. However, the retrospective nature of these trials, the small number of patients enrolled, the use of different chemotherapy regimens and the presence of conflicting data, question the reliability of DPD expression as a predictive factor in this context. Smorenburg et al ${ }^{[67]}$ realized the only prospective study in which the expression of TS and DPD (in 53 patients with advanced colorectal cancer), led the choice of the first line chemotherapy. They proved that patients who had low TS and low DPD levels have higher response rate to 5-FU/LV compared with patients treated with non 5-FU containing regimen. The most relevant limitation of this study is that the population was divided into two groups with different markers and treated with different chemotherapy; so this does not allow a comparison between the groups and does not confirm the DPD expression predictive role.

The B-CAST study is a multicenter, prospective cohort trial aimed to identify a correlation between tumor biomarkers expression, including DPD expression determined by RT-PCR, and outcome benefit from adjuvant treatment with different 5 -FU based regimens in a population with stage III colon cancer: preliminary results of the study are actually unavailable, but we believe that this study might contribute to clarify the role of DPD as a predictive marker in this setting, owing to the large number of patients (2128) enrolled. 
Table 2. Dihydropyrimidine dehydrogenase expression correlation with response and outcome in patients with advanced colorectal cancer receiving fluoropyrimidine-based chemotherapy

\begin{tabular}{|c|c|c|c|c|c|c|c|}
\hline Study (ref.) & Treatment setting & Regimen & Patients $(n)$ & Method & Better response & Longer TTP/PFS & Longer OS \\
\hline Salonga et al. ${ }^{[52]}$ & Advanced & $5-F U+L V$ & 33 & RT-PCR & Low DPD exp. & & \\
\hline Meropol et al..$^{[54]}$ & Advanced & CAPIRI & 67 & RT-PCR & NS & & \\
\hline Vallböhmer et al. ${ }^{[51]}$ & Advanced & CAP-based & 37 & RT-PCR & Low DPD exp. & Low DPD exp. & \\
\hline Yanagisawa et al. ${ }^{[53]}$ & Advanced & $5-F U+L V$ & 21 & RT-PCR & NS & & Low DPD exp. \\
\hline Gustavsson et al. ${ }^{[56]}$ & Advanced & 5-FU-based & 144 & RT-PCR & & Low DPD exp. & \\
\hline Koopman et al. ${ }^{[55]}$ & Advanced & CAPIRI & 283 & $\mathrm{IHC}$ & & Low DPD exp. & Low DPD exp. \\
\hline
\end{tabular}

CAP: capecitabine; CAPIRI: capecitabine + irinotecan; DPD: dihydropyrimidine dehydrogenase; ELISA: enzyme-linked; Exp: expression; IHC: immununohistochemical analysis; LV: leucovorin; NS: not significant; OS: overall survival; PFS: progression-free survival; RT-PCR: reverse transcriptase PCR; TTP: time to progression; 5-FU: 5-fluorouracil

Table 3. Dihydropyrimidine dehydrogenase expression correlation with response and outcome in patients with resected colorectal cancer receiving fluoropyrimidine-based chemotherapy

\begin{tabular}{|c|c|c|c|c|c|c|c|}
\hline Study (ref.) & Treatment setting & Regimen & Patients $(n)$ & Method & Better response & Longer DFS & Longer OS \\
\hline Kornmann et al. $^{[65]}$ & Adjuvant & 5-fu-based & 348 & RT-PCR & & NS & NS \\
\hline Ikeguchi et al. ${ }^{[66]}$ & Adjuvant & $5-\mathrm{FU}$ & 100 & ELISA & & & NS \\
\hline Kornmann et al. ${ }^{[63]}$ & Adjuvant & 5-FU-based & 295 & RT-PCR & & & Low DPD exp. \\
\hline Oi et al. ${ }^{[58]}$ & Adjuvant & 5-FU-based & 64 & $\mathrm{IHC}$ & & Low DPD exp. & \\
\hline Westra et $a I^{[64]}$ & Adjuvant & 5-FU-based & 341 & $\mathrm{IHC}$ & & - & \\
\hline Ciaparrone et al. ${ }^{[57]}$ & Adjuvant & $5-F U+L V$ & 62 & $\mathrm{IHC}$ & & Low DPD exp. & Low DPD exp. \\
\hline Lassman et al. ${ }^{[59]}$ & Adjuvant & 5-FU-based & 52 & RT-PCR & & Low DPD exp. & \\
\hline Hotta et al. ${ }^{[60]}$ & Adjuvant & $5-F U+L V$ & 22 & ELISA & & Low DPD exp. & NS \\
\hline Jensen et $a / .{ }^{[61]}$ & Adjuvant & $5-F U+I V$ & 303 & $\mathrm{IHC}$ & & Low DPD exp. & Low DPD exp. \\
\hline Soong et al. ${ }^{[62]}$ & Adjuvant & $5-F U+L V$ & 123 & $\mathrm{IHC}$ & & & NS \\
\hline
\end{tabular}

DFS: disease-free survival; DPD: dihydropyrimidine dehydrogenase; ELISA: enzyme-linked immunosorbed assay; Exp: expression; IHC: immununohistochemical analysis; IV: isovorin; LV: leucovorin; NS: not significant; OS: overall survival; RT-PCR: reverse transcriptase PCR; 5-FU: 5- fluorouracil; -: no positive correlation

\section{Gastric cancer}

5-FU based chemotherapy is commonly used for both palliative treatment of metastatic gastric cancer (GC) and adjuvant therapy in patients who underwent surgery. Therefore the role of DPD expression as a predictive biomarker for chemotherapy sensitivity and for outcome in patients treated with 5-FU has been widely investigated in this setting.

Toriumi et al ${ }^{[68]}$ reported a statistically significant correlation, in vitro, between sensitivity to 5-FU therapy and DPD expression: in particular, cells that exhibited high expression of DPD mRNA were resistant to 5-FU; a weak inverse correlation between DPD level and sensitivity to 5'-deoxy-5-fluorouridine (5'-DFUR) in vitro was also reported by Terashima et al. ${ }^{[69]}$. Some other studies also showed high DPD expressions levels in 5-FU resistant cell lines ${ }^{[70,71]}$ while DPD mRNA expression did not correlate with 5-FU sensitivity in a study conducted by Kodera et al. ${ }^{[72]}$. In vivo studies by Nishina et al ${ }^{[73]}$ and by Koizumi et al ${ }^{[74]}$, conducted in a population affected by advanced GC, suggested that patients with high thymidine phosphorylase (TP) and low DPD expression are more sensitive to $5^{\prime}$-DFUR ${ }^{[73]}$ and capecitabine ${ }^{[74]}$ respectively. However a low DPD association has been associated with worst response rate in patients treated with $\mathrm{S}-1^{[75]}$, a chemotherapy containing 5-chloro-2,4-dihydroxypiridine (CDHP) with anti-DPD activity widely used in Japan, and other studies demonstrated not statistically significant results with the same agent ${ }^{[76]}$.

Similarly to colon cancer patients, also in GC, the predictive role of DPD was investigated in both metastatic and adjuvant setting.

In adjuvant setting a longer survival was reported in patients treated with 5'-DFUR chemotherapy showing high TP to DPD ratio ${ }^{[69]}$. Opposite results were reached by other two studies, where low intratumoral DPD 
expression was correlated with worse $\mathrm{DFS}^{[77]}$ and $\mathrm{OS}^{[78]}$ in patients who received S-1 adjuvant therapy.

A study conducted by Grau et al. ${ }^{[79]}$ investigated the role of genetic variations in DPYD in place of looking at DPD expression, analyzing the prognostic value of genetic single nucleotide polymorphisms (SNPs) of intratumoral DPYD and CDA (cytidine-deaminase enzyme, which can influence the activity of TS) in patients with GC treated with adjuvant fluoropyrimidine (tegafur). SNP of DPYD1 (A/G; Ile453Val) was associated with better survival while the SNP (C/T; Arg29Cys) of DPYD2 showed a benefit in terms of relapse and survival if associated with the polymorphism of CDA (A/C; Lys27Gln $)^{[79]}$.

Conflicting results are reported also in the metastatic setting. Some studies have demonstrated that high DPD expression can be a predictor of poor survival in patients treated with $\mathrm{S}-1^{[8,8,1]}$ and $5-\mathrm{FU}^{[68]}$, while a study conducted by Nishina et al. ${ }^{[73]}$ highlighted a connection between high TP to DPD ratio and longer OS in patients treated with 5-DFUR chemotherapy. At the same time various studies reported the absence of statistically significant impact of DPD value on survival in patients treated with S- ${ }^{[75,76,82]}$ and $5-\mathrm{FU}^{[75,83]}$.

Given the controversial results previously reported, in 2017, Zhang et al. ${ }^{[84]}$ performed a meta-analysis aimed to assess the potential impact of intratumoral DPD expression level on chemotherapy sensitivity and long-term survival for GC. The meta-analysis confirmed a correlation between high DPD expression and 5-FU activity, whereas long-term survival was not significantly different.

It should be noted that, while studies that assessed the activity of the chemotherapy regimens were conducted mainly by using 5-FU, studies that contributed in the meta-analysis to survival outcomes were those where S-1 was used more frequently. S-1 is a combined drug consisting of tegafur and CDHP, a chemotherapeutic agent characterized by DPD inhibitor activity; proof of this activity is the absence of hand-foot syndrome due to the lower concentration of 5-FU degradation products in patients treated with S-1 unlike patients treated with 5-FU or capecitabine. Thus, an enhancement of the antitumor effect would be expected in patients with high intratumoral DPD expression in comparison with patients treated with 5-FU, potentially invalidating the prognostic role of DPD expression level. While part of the previously cited studies confirm this hypothesis highlighting no significant prognostic difference between patients with high or low DPD expression or a worse prognosis in case of patients with low DPD levels treated with S-1, other studies provide opposite results. In fact, even if CDHP inhibiting activity in vitro is established, the degree of intratumoral DPD activity inhibited by this agent in clinical setting is unknown.

Furthermore, the retrospective design of these studies, coupled with the lack of a reproducible assay for DPD expression, represents a severe limitation that could explain the lack of consistent data. Moreover, due to the fact that the majority of the studies included in the meta-analysis were conducted in Japan, due to the aforementioned described ethnic differences in terms of DPD expression, definitive conclusions concerning the association between DPD and sensitivity to chemotherapy can not apply to all populations.

\section{Other cancer types}

The role of DPD expression as a prognostic factor has been mainly investigated in CRC and GC as previously described, however the widely use of 5-FU based chemotherapy has encouraged researchers to examine its value as a predictive and prognostic factor in other tumor types. Some studies showed a survival benefit in patients with low DPD expression treated with adjuvant $\mathrm{S}-1^{\left[{ }^{85]}\right.}$ or $5-\mathrm{FU}^{[86,87]}$ after the resection of pancreatic cancer. However, in a study conducted by Murakawa et al ${ }^{[88]}$, no significant difference in terms of 3-year OS following surgery was reported between DPD-postive and DPD-negative expression groups in patients treated with S-1 chemotherapy. In other studies which enrolled a small number of pancreatic cancer patients adjuvantly treated with 5-FU based chemotherapy, low TP/DPD ratio was significantly correlated with longer survival ${ }^{[8,90]}$. 
In regard to head and neck cancer, intratumoral overexpression of DPD has been correlated with resistance to 5 -FU-based chemotherapy in metastatic ${ }^{[91]}$ and neoadjuvant setting ${ }^{[92]}$. In contrast, in a recent study conducted by Hasegawa et al. ${ }^{[93]}$, higher DPD expression was predictive of better response to induction chemotherapy with 5-FU/cisplatin.

Few studies has been carried out in breast cancer patients treated with FU-based treatment. While some studies assessed a role of low DPD expression as a predictive factor for chemosensitivity ${ }^{[94]}$ and for DFS in adjuvant setting ${ }^{[95]}$, other ones did not found a significant difference between high and low DPD groups in terms of DFS in adjuvantly treated patients ${ }^{[96,97]}$ and OS in metastatic ones ${ }^{[98]}$. A recent study conducted by Qin et al. ${ }^{[99]}$ was the first aimed to investigate the prognostic role of DPYD polymorphisms in breast cancer. They demonstrated that DPYD c.1627A $>$ G AG/GG polymorphism, detected from tumor tissue, was associated with poor OS and PFS in non-luminal patients treated with fluoropyrimidine-based chemotherapy $^{[99]}$.

Conflicting data are also reported in lung cancer patients, with DPD expression pointed out as either related $^{[100]}$ or not significantly associated with prognosis ${ }^{[101]}$.

In addition to the described limitations of the studies regarding the potential predictive and prognostic role of DPD expression in CRC and GC, the relative small number of investigations focused on different tumor types makes a hypothetical consensus unreachable at the moment.

\section{CPIC $^{\circledR}$ AND DOSE ADJUSTMENT}

Clinical Pharmacogenetics Implementation Consortium (CPIC $)$ is an International Consortium establishing guidelines interested in facilitating use of pharmacogenetic tests for patient care; it helps clinicians interpreting genetic test results and ensuring a safer drug delivery to the patient ${ }^{[102]}$.

In the CPIC Guidelines for DPYD genotype and fluoropyrimidine dosing it is summarized the relationship between DPYD polymorphism deficiency and the dose-adjustment of 5-FU based chemotherapy.

In the 2013 edition, initial dose reduction was recommended only for the c.2846A>T, c.1679T>G and $D P Y D^{\star} 2 \mathrm{~A}$ polymorphisms; if after two chemotherapy cycles the treatment proved to be safe, individual dose can be titrated to have maximum safe drug concentration in every patient ${ }^{[49]}$.

In the 2017 update all the four DPYD variants discussed above have been identified as relevant due to their frequency, their impact on enzyme function and clinical toxicity. Among them, $D P Y D^{\star} 2 \mathrm{~A}$ and $D P Y D^{\star} 13$ have the most dangerous consequences on DPD enzyme activity; c.2846A $>$ T and c.1129-5923C $>\mathrm{G}$ variants cause a moderate activity reduction.

The most common decreased function DPD variant in Europe is HapB3 with c.1129-5923C $>\mathrm{G}$ (4.7\%), followed by c. $1905+1 \mathrm{G}>\mathrm{A}(1.6 \%)$ and c.2846A $>\mathrm{T}(0.7 \%)$. Adding all the four variants $7 \%$ of Europeans carry one decreased function DPYD allele. All the other allele frequency are $\operatorname{rarer}^{[31]}$.

In Table 4 are shown correlations between DPD phenotype and DPYD genotype, assessed only for few variants, because the functional impact of rarer polymorphism has been only evaluated in vitro. The DPD phenotype is assigned using a gene activity-score (DPYD-AS): carriers of two no functional alleles are classified as DPYD poor metabolizers (DPYD-AS:0); carriers of one no function or decreased function are $D P Y D$ intermediate metabolizers (DPYD-AS:1 or 1.5$)$ and those with normal function alleles are DPYD normal metabolizers (DPYD-AS:2). 
Table 4. Assignment of likely DPD phenotypes based on DPYD genotypes

\begin{tabular}{|c|c|c|c|}
\hline Likely phenotype & Activity score & Genotypes & Examples of Genotypes \\
\hline Normal metabolizer & 2 & Carriers of two normal function alleles & c. $[=] ;[=]$, c. $[85 \mathrm{~T}>\mathrm{C}] ;[=]$, c. $[1627 \mathrm{~A}>\mathrm{G}] ;[\mathrm{i}=]$ \\
\hline Intermediate metabolizer & 1 or 1.5 & $\begin{array}{l}\text { Carriers of one normal function allele } \\
\text { plus one no function allele or one } \\
\text { decreased function allele, or carriers of } \\
\text { two decreased function alleles }\end{array}$ & $\begin{array}{l}\text { c. }[190511 \mathrm{G}>\mathrm{A}] ;[=], \mathrm{c} .[1679 \mathrm{~T}>\mathrm{G}] ;[=] \\
\text { c. }[2846 \mathrm{~A}>\mathrm{T}] ;[=] ; \mathrm{c} .[1129-5923 \mathrm{C}>\mathrm{G}] ; \\
{[=] ; \mathrm{c} .[1129-5923 \mathrm{C}>\mathrm{G}] ;[1129-5923 \mathrm{C}>\mathrm{G}]} \\
\text { c. }[2846 \mathrm{~A}>\mathrm{T}] ;[2846 \mathrm{~A}>\mathrm{T}]\end{array}$ \\
\hline Poor metabolizer & 0 or 0.5 & $\begin{array}{l}\text { Carriers of two no function alleles or } \\
\text { carriers of one no function plus one } \\
\text { decreased function allele }\end{array}$ & $\begin{array}{l}\text { c. }[190511 G>A] ;[190511 G>A] \\
\text { c. }[1679 T>G] ;[1679 T>G] \text {, } \\
\text { c. }[190511 G>A] ;[2846 A>T] \text { c. }[190511 G>A] ; \\
{[1129-5923 C>G]}\end{array}$ \\
\hline
\end{tabular}

Table 5. Recommended dosing of Fluoropyrimidines by DPD phenotype

\begin{tabular}{|c|c|c|c|}
\hline Phenotype & Clinical consequences & Dosing recommendations & $\begin{array}{l}\text { Classification of } \\
\text { recommendations }\end{array}$ \\
\hline $\begin{array}{l}\text { DPYD normal } \\
\text { metabolizer }\end{array}$ & Normal DPD activity. & Label-recommended dosage and administration. & Strong \\
\hline $\begin{array}{l}D P Y D \text { intermediate } \\
\text { metabolizer }\end{array}$ & $\begin{array}{l}\text { Decreased DPD activity and } \\
\text { increased risk for drug toxicity. }\end{array}$ & $\begin{array}{l}\text { Reduce starting dose based on activity score followed } \\
\text { by titration of dose based on toxicity or therapeutic drug } \\
\text { monitoring (if available). } \\
\text { Activity score 1: Reduce dose by } 50 \% \\
\text { Activity score 1.5: Reduce dose by } 25 \% \text { to } 50 \%\end{array}$ & $\begin{array}{l}\text { Activity score 1: } \\
\text { strong } \\
\text { Activity score 1.5: } \\
\text { moderate }\end{array}$ \\
\hline $\begin{array}{l}\text { DPYD poor } \\
\text { metabolizer }\end{array}$ & $\begin{array}{l}\text { Complete DPD deficiency and } \\
\text { increased risk for drug toxicity }\end{array}$ & $\begin{array}{l}\text { Activity score 0.5:avoid use of fluoropyrimidines. If there are } \\
\text { not alternatives agents, 5-FU should be administered at a } \\
\text { strongly reduced dose. } \\
\text { Activity score 0: Avoid fluoropyrimidines }\end{array}$ & Strong \\
\hline
\end{tabular}

Table 5 contains the genetic-based recommendations for fluoropyrimidines-dose using the calculated $D P Y D$ activity score (DPYD-AS).

Patients heterozygous for DPYD decreased/no function variants should receive reduced starting dose because of partial enzyme activity. In particular heterozygous carriers of $D P Y D^{*} 2 \mathrm{~A}$ treated with $50 \%$ dose reduction showed a rate of severe toxicity comparable to non carriers (DPYD-AS:1) ${ }^{[38]}$. In patients with c. $2846 \mathrm{~A}>\mathrm{T}$ heterozygous polymorphism a retrospective study established that the capecitabine starting dose was reduced by $25 \%$ compared to non carriers ${ }^{[44]}$, just like the carriers of $\mathrm{c} .1236 \mathrm{G}>\mathrm{A}^{[103]}$. So the finally deduction is that patients carriers of DYD-AS:1.5 can tolerate higher doses than patients carriers of no function variants (DPYD-AS:1). In patients with decreasing function other circumstance should be considered to determine the initial dose-reduction, 50\% starting dose followed by dose titration or only a $25 \%$ dose reduction.

If the first two cycles are well tolerated, to maintain effectiveness dose could be increased in subsequent cycles, given that some patients, even if carriers of no function or less function variants, tolerate normal dose of 5-FU. Similarly, if no tolerated, dose should be decreased.

In DPYD poor metabolizers ( $D P Y D$-AS: 0.5 or 0 ) it is strongly recommended to avoid 5 -FU based chemotherapy. However, if there are no other options, 5-FU regimen at a heavily reduced dose in association with early drug monitoring may be considered for DPYD-AS:0.5. It should be noted that no reports of successful administration of a low dose of 5-FU in DPD poor metabolizers are available. It is estimated that a dose-reduction of $75 \%$ would be required. The CPIC defines as poor metabolizer only patients with two no-functional alleles; this score could be considered poor sensitive and high specific. We speculate that this approach aims to avoid false positive results in order not to deprive patients of 5-FU, given its primary role in solid tumors treatment. 


\section{DPD AND COSTS}

DPYD mutations tests are not routinely performed because of the initial costing concerns of the tests and the absence of clear dose reduction guidelines in patients who have a deficit of DPD in prospective tests ${ }^{[104]}$. Considering the ever-increasing number of patients that will require fluoropyrimidine-based chemotherapy, concerns regarding also the usefulness and costs of prospective testing have arisen.

Furthermore, the absence of a mutation does not guarantee the absence of serious toxicity. Conversely, the potential benefit of prospective identification of DPYD mutations is that, careful monitoring and gradual dose escalation, may allow patients with DPD deficiency to receive fluoropyrimidine chemotherapy safely ${ }^{[6,105]}$.

Murphy et al. ${ }^{[106]}$ have shown that the cost of managing patients with severe toxicity to chemotherapy and with DPYD reactive test is higher than the cost of the prospective test on each new patient who initiates fluoropyrimidine chemotherapy. These results therefore showed that tests for the detection of $D P Y D$ mutations in the study population would be associated with significant cost savings. Another notrandomized study ${ }^{[107]}$ showed that prospective tests for DPD deficiency in patients receiving 5FU-based chemotherapy for CRC could be a cost-effective strategy.

Prospective identification of patients with a DPYD mutation, associated with a dose reduction from the start of therapy, can avoid chemotherapy-related toxicity and improve quality of life. In clinical practice, it is therefore reasonable to reduce the doses appropriately for the best characterized polymorphisms, avoiding testing polymorphisms with a still undefined meaning. In patients with these polymorphisms, greater vigilance may be suggested without dose reductions from the start of therapy. Routine prospective tests are therefore economically viable even if further research and clear dose reduction recommendations are needed. The DPYD test has the potential to prevent premature cessation of potentially curative therapy for patients with deficiencies ${ }^{[106,107]}$.

\section{CONCLUSIONS}

Fluoropyrimidines still remain a class of pivotal drugs in the treatment of solid tumors. In this context the DPD enzyme plays an essential role as responsible for the inactivation of 5-FU. To date, many polymorphisms of the gene coding for this enzyme are known, determining various degrees of toxicity in patients who are treated with these drugs. The studies focused on 4 of these genotypes, those with the highest reported frequency in the population and those with a greater clinical impact: $D P Y D^{\star} 2 \mathrm{~A}$, c. $1679 \mathrm{~T}>\mathrm{G}, \mathrm{c} .2846 \mathrm{~A}>\mathrm{T}$ and c.1236G $>\mathrm{A}$. These polymorphisms differ in the degree of toxicity and therefore each patient should receive dosage of these drugs that is based on the activity of this enzyme.

Furthermore, the expression of DPD should have profound impact in terms of prognosis and as predictor of effectiveness of these drugs. Speaking from a prognostic point of view, the presence of high intratumoral DPD expression should be associated with chemoresistance and worst outcome. The validation of this assumption would guide clinicians in everyday practice, encouraging, for instance, the use of 5-FU-based adjuvant therapy in patients at intermediate risk of recurrence after surgical resection or, moreover, the use of fluoropyrimidine-including treatment in metastatic setting, in presence of low DPD expression. On other hand, from a predictive point of view, due to the paramount importance of 5-FU-based chemotherapy in CRC, low DPD expression should be associated to better response/outcome in patients treated with fluoropyrimidine. This matter has been assessed in a series of studies in the setting of CRC. However, these studies are affected by various limitations: most of these studies are retrospective analyses and the majority of them is characterized by small sample size. We must mention also the lack of a standardized method of measurement of DPD, as it has been carried out in various papers through different ways (RT-PCR, IHC 
or ELISA), and the absence of a defined cut-off which could differentiate high from low DPD expression. Furthermore, there was no standardization even for chemotherapy regimens, given the use of different types and doses of fluoropyrimidine and the combinations with different chemotherapeutic agents such as cisplatin and oxaliplatin, which could have highly influenced reported results. Therefore, even if there seems to be a trend supporting the correlation between DPD expression and response/survival in patients treated with fluoropyrimidine, no definitive conclusions can be pointed out, considering also that some studies showed not statistically significant or even opposite results. The same statements could be referred to GC setting, which is also affected by limitations of ethnicity (almost all studies were conducted in Japan) and possible results distorsions due to the wide use of S-1 (in Japanese population), whose DPD inhibiting activity may invalidate possible statement concerning the role of DPD expression as a predictive factor.

Thus the debate on the intratumoral DPD expression as a potential predictive and prognostic marker in fluoropyrimidine treated patients, is still open and it is crucial to analyze its role through prospective studies ${ }^{[108]}$, standardized for DPD expression assessment method and chemoterapeutic regimen used. Moreover, given the complexity of 5-FU pharmacokinetic, it would be useful to integrate future prospective studies with the evaluation of the other enzymes involved in 5-FU metabolic pathway.

\section{DECLARATIONS}

\section{Authors' contributions}

All authors contributed equally to the article.

\section{Availability of data and materials}

Not applicable.

\section{Financial support and sponsorship}

None.

\section{Conflicts of interest}

All authors declared that there are no conflicts of interest.

\section{Ethical approval and consent to participate}

Not applicable.

\section{Consent for publication}

Not applicable.

\section{Copyright}

(c) The Author(s) 2019.

\section{REFERENCES}

1. Ezzeldin H, Diasio R. Dihydropyrimidine dehydrogenase deficiency, a pharmacogenetic syndrome associated with potentially lifethreatening toxicity following 5-fluorouracil administration. Clin Colorectal Cancer 2004;4:181-9.

2. Scrip's Cancer Chemotherapy Report. Scrip World Pharmaceutical News. London: PJB Publications Ltd 2002.

3. Loriot MA, Ciccolini J, Thomas F, Barin-Le-Guellec C, Royer B, et al. Dihydropyrimidine déhydrogenase (DPD) deficiency screening and securing of fluoropyrimidine-based chemotherapies: Update and recommendations of the French GPCO-Unicancer and RNPGx networks. Bull Cancer 2018; 105:397-407.

4. Heggie GD, Sommadossi JP, Cross DS, Huster WJ, Diasio RB. Clinical pharmacokinetics of 5-fluorouracil and its metabolites in plasma, urine, and bile. Cancer Res 1987;47: 2203-6.

5. Van Cutsem E, Twelves C, Cassidy J, Allman D, Bajetta E, et al. Oral capecitabine compared with intravenous fluorouracil plus leucovorin in patients with metastatic colorectal cancer: results of a large phase III study. J Clin Oncol 2001;19:4097-106. 
6. Rosmarin D, Palles C, Pagnamenta A, Kaur K, Pita G, et al. A candidate gene study of capecitabine-related toxicity in colorectal cancer identifies new toxicity variants at DPYD and a putative role for ENOSF1 rather than TYMS. Gut 2015;64:111-20.

7. Saltz LB, Niedzwiecki D, Hollis D, Goldberg RM, Hantel A, et al. Irinotecan fluorouracil plus leucovorin is not superior to fluorouracil plus leucovorin alone as adjuvant treatment for stage III colon cancer: results of CALGB 89803. J Clin Oncol 2007; 25:3456-61.

8. Harris BE, Song R, Soong SJ, Diaso RB. Relationship between dihydropyrimidine dehydrogenase activity and plasma 5-fluorouracil levels with evidence for circadian variation of enzyme activity and plasma drug levels in cancer patients receiving 5 -fluorouracil by protracted continuous infusion. Cancer Res 1990;50:197-201.

9. Grem JL, Yee LK, Venzon D, Takimoto CH, Allegra CJ. Inter- and intra-individual variation in dihydropyrimidine dehydrogenase activity in peripheral blood mononuclear cells. Cancer Chemother Pharmacol 1997;40:117-25.

10. Ogura K, Ohnuma T, Minamide Y, Mizuno A, Nishiyama A, et al. Dihydropyrimidine dehydrogenase activity in 150 healthy Japanese volunteers and identification of novel mutations. Clin Cancer Res 2005;11:5104-111.

11. Morsman JM, Sludden J, Ameyaw MM, Githang J, Indalo A, et al. Evaluation of dihydropyrimidine dehydrogenase activity in Southwest Asian, Kenyan and Ghanaian populations. Br J Clin Pharmacol 2000;50:269-72.

12. Yamaguchi K, Arai Y, Kanda Y, Akagi K. Germline mutation of dihydropyrimidine dehydrogenase gene among a Japanese population in relation to toxicity to 5-fluorouracil. Jpn J Cancer Res 2001;92:337-42.

13. Diasio RB, Harris BE. Clinical pharmacology of 5-fluorouracil. Clin Pharmacokinet 1989;16:215-37.

14. Longley DB, Harkin DP, Johnston PG. 5-fluorouracil: mechanisms of action and clinical strategies. Nat Rev Cancer 2003;3:330-8.

15. Johnson MR, Diasio RB. Importance of dihydropyrimidine dehydrogenase (DPD) deficiency in patients exhibiting toxicity following treatment with 5-fluorouracil. Adv Enzyme Regul 2001;41:151-7.

16. Mattison LK, Fourie J, Desmond RA, Modak A, Saif MW, et al. Increased prevalence of dihydropyrimidine dehydrogenase deficiency in African-Americans compared with Caucasians. Clin Cancer Res 2006;12:5491-5.

17. Lu Z, Zhang R, Diasio RB. Dihydropyrimidine dehydrogenase activity in human peripheral blood mononuclear cells and liver: population characteristics, newly identified deficient patients, and clinical implication in 5-fluorouracil chemotherapy. Cancer Res 1993;53:5433-8.

18. Etienne MC, Lagrange JL, Dassonville O, Fleming R, Thyss A, et al. Population study of dihydropyrimidine dehydrogenase in cancer patients. J ClinOncol 1994;12:2248-53.

19. Amstutz U, Froehlich TK, Largiadèr CR. Dihydropyrimidine dehydrogenase gene as a major predictor of severe 5-fluorouracil toxicity. Pharmacogenomics 2011;12:1321-36.

20. Morel A, Boisdron-Celle M, Fey L, Soulie P, Craipeau MC, et al. Clinical relevance of different dihydropyrimidine dehydrogenase gene single nucleotide polymorphisms on 5-fluorouracil tolerance. Mol Cancer Ther 2006;5:2895-904.

21. Van Kuilenburg AB, Haasjes J, Richel DJ, Zoetekouw L, Van Lenthe H, et al. Clinical implications of dihydropyrimidine dehydrogenase (DPD) deficiency in patients with severe 5-fluorouracil-associated toxicity: identification of new mutations in the DPD gene. Clin Cancer Res 2000;6:4705-12.

22. Milano G, Etienne MC, Pierrefite V, Barberi-Heyob M, Deporte-Fety R, et al. Dihydropyrimidine dehydrogenase deficiency and fluorouracilrelated toxicity. Br J Cancer 1999;79:627-30.

23. Van Kuilenburg AB, Meinsma R, Zoetekouw L, Van Gennip AH. Increased risk of grade IV neutropenia after administration of 5 -fluorouracil due to a dihydropyrimidine dehydrogenase deficiency: high prevalence of the IVS14+1G $>$ A mutation. Int J Cancer 2002;101:253-8.

24. Wei X, Elizondo G, Sapone A, McLeod HL, Raunio H, et al. Characterization of the human dihydropyrimidine dehydrogenase gene. Genomics 1998;51:391-400.

25. Offer SM, Diasio RB. Is it finally time for a personalized medicine approach for fluorouracil-based therapies? J Clin Oncol 2016;34:205-7.

26. Deenen MJ, Meulendijks D, Cats A, Sechterberger MK, Severens JL, et al. Upfront genotyping of DPYD*2A to individualize fluoropyrimidine therapy: a safety and cost analysis. J Clin Oncol 2016;34:227-34.

27. Launay M, Ciccolini J, Rodallec A, Fournel C, Duffaud F, et al. Upfront DPD Deficiency Detection to Secure 5-FU Administration: Part 1 - Where Do We Stand? Clin Cancer Drugs 2017;4:74-81.

28. Siddiqui NS, Purvey S, Hamal R, Zhang L, Diasio RB, et al. Dihydropyrimidine dehydrogenase gene (DPYD) polymorphism among pts with 5-FU/capecitabine (CAP)-related adverse events (AEs): Experience of 2 decades. J Clin Oncol 2018;36:2576.

29. Van Staveren MC, Guchelaar HJ, Van Kuilenburg ABP, Gelderblom H, Maring JG. Evaluation of predictive tests for screening for dihydropyrimidine dehydrogenase deficiency. Pharmacogenomics J 2013;13:389-95.

30. Launay M, Dahan L, Duval M, Rodallec A, Milano G, et al. Beating the odds: efficacy and toxicity of dihydropyrimidine dehydrogenasedriven adaptive dosing of 5-FU in patients with digestive cancer. Br J Clin Pharmacol 2016;81:124-30.

31. Amstutz U, Henricks LM, Offer SM, Barbarino J, Schellens JHM, et al. Clinical Pharmacogenetics Implementation Consortium (CPIC) Guideline for Dihydropyrimidine Dehydrogenase Genotype and Fluoropyrimidine Dosing: 2017 Update. Clin Pharmacol Ther 2018;103:210-6.

32. Lee AM, Shi Q, Pavey E, Alberts SR, Sargent DJ, et al. DPYD variants as predictors of 5-fluorouracil toxicity in adjuvant colon cancer treatment (NCCTG N0147). J Natl Cancer Inst 2014;106:1-12.

33. Meulendijks D, Cats A, Beijnen JH, Schellens JH. Improving safety of fluoropyrimidine chemotherapy by individualizing treatment based on dihydropyrimidine dehydrogenase activity - ready for clinical practice? Cancer Treat Rev 2016;50:23-34.

34. Henricks LM, Lunenburg CA, Meulendijks D, Gelderblom H, Cats A, et al. Translating DPYD genotype into DPD phenotype: using the DPYD gene activity score. Pharmacogenomics 2015;16:1277-86.

35. Van Kuilenburg AB, Muller EW, Haasjes J, Meinsma R, Zoetekouw L, et al. Lethal outcome of a patient with a complete 
dihydropyrimidine dehydrogenase (DPD) deficiency after administration of 5-fluorouracil: frequency of the common IVS14 + 1G>A mutation causing DPD deficiency. Clin Cancer Res 2001;7:1149-53.

36. Van Kuilenburg AB, Vreken P, Beex LV, Meinsma R, Van Lenthe H, et al. Heterozygosity for a point mutation in an invariant splice donor site of dihydropyrimidine dehydrogenase and severe 5-fluorouracil related toxicity. Eur J Cancer 1997;33:2258-64.

37. Offer SM, Wegner NJ, Fossum C, Wang K, Diasio RB. Phenotypic profiling of DPYD variations relevant to 5-fluorouracil sensitivity using real-time cellular analysis and in vitro measurement of enzyme activity. Cancer Res 2013;73:1958-68.

38. Johnson MR, Hageboutros A, Wang K, High L, Smith JB, et al. Life-threatening toxicity in a dihydropyrimidine dehydrogenase-deficient patient after treatment with topical 5-fluorouracil. Clin Cancer Res 1999;5:2006-11.

39. Seck K, Riemer S, Kates R, Ullrich T, Lutz V, et al. Analysis of the DPYD gene implicated in 5-fluorouracil catabolism in a cohort of Caucasian individuals. Clin Cancer Res 2005;11:5886-92.

40. Caudle KE, Thorn CF, Klein TE, Swen JJ, McLeod HL, et al. Clinical Pharmacogenetics Implementation Consortium guidelines for dihydropyrimidine dehydrogenase genotype and fluoropyrimidine dosing. Clin Pharmacol Ther 2013;94:640-5.

41. Offer SM, Fossum CC, Wegner NJ, Stuflesser AJ, Butterfield GL, et al. Comparative functional analysis of DPYD variants of potential clinical relevance to dihydropyrimidine dehydrogenase activity. Cancer Res 2014;74:2545-54.

42. Amstutz U, Farese S, Aebi S, Largiadèr CR. Dihydropyrimidine dehydrogenase gene variation and severe 5-fluorouracil toxicity: a haplotype assessment. Pharmacogenomics 2009;10:931-44.

43. Van Kuilenburg AB, Meijer J, Mul AN, Meinsma R, Schmid V, et al. Intragenic deletions and a deep intronic mutation affecting premRNA splicing in the dihydropyrimidine dehydrogenase gene as novel mechanisms causing 5-fluorouracil toxicity. Hum Genet 2010;128:529-38.

44. Deenen MJ, Tol J, Burylo AM, Doodeman VD, de Boer A, et al. Relationship between single nucleotide polymorphisms and haplotypes in DPYD and toxicity and efficacy of capecitabine in advanced colorectal cancer. Clin Cancer Res 2011;17:3455-68.

45. Froehlich TK, Amstutz U, Aebi S, Joerger M, Largiadèr CR. Clinical importance of risk variants in the dihydropyrimidine dehydrogenase gene for the prediction of early-onset fluoropyrimidine toxicity. Int J Cancer 2015;136:730-9.

46. Sistonen J, Büchel B, Froehlich TK, Kummer D, Fontana S, et al. Predicting 5-fluorouracil toxicity: DPD genotype and 5,6-dihydrouracil:uracil ratio. Pharmacogenomics 2014;15:1653-66.

47. Meulendijks D, Henricks LM, van Kuilenburg AB, Jacobs BA, Aliev A, et al. Patients homozygous for DPYD c.1129-5923C >G/ haplotype B3 have partial DPD deficiency and require a dose reduction when treated with fluoropyrimidines. Cancer Chemother Pharmacol 2016;78:875-80.

48. Meulendijks D, Henricks LM, Sonke GS, Deenen MJ, Froehlich TK, et al. Clinical relevance of DPYD variants c.1679T >G, c.1236G >A/ HapB3, and c. $1601 \mathrm{G}>\mathrm{A}$ as predictors of severe fluoropyrimidine-associated toxicity: a systematic review and meta-analysis of individual patient data. Lancet Oncol 2015;16:1639-50.

49. Henricks LM, Opdam FL, Beijnen JH, Cats A, Schellens JHM. DPYD genotype-guided dose individualization to improve patient safety of fluoropyrimidine therapy: call for a drug label update. Ann Oncol 2017;28:2915-22.

50. Henricks LM, Lunenburg CATC, de Man FM, Meulendijks D, Frederix GWJ, et al. DPYD genotype-guided dose individualisation of fluoropyrimidine therapy in patients with cancer: a prospective safety analysis. Lancet Oncol 2018;19:1459-67.

51. Vallböhmer D, Yang DY, Kuramochi H, Shimizu D, Danenberg KD, et al. DPD is a molecular determinant of capecitabine efficacy in colorectal cancer. Int J Oncol 2007;31:413-8.

52. Salonga D, Danenberg KD, Johnson M, Metzeger R, Groshen S, et al. Colorectal tumors responding to 5-fluorouracil have low gene expression levels of dihydropyrimidine dehydrogenase, thymidylate synthase, and thymidine phosphorylase. Clin Cancer Res 2000;6:1322-7.

53. Yanagisawa Y, Maruta F, Iinuma N, Ishizone S, Koide N, et al. Modified irinotecan/5FU/leucovorin therapy in advanced colorectal cancer and predicting therapeutic efficacy by expression of tumorrelated enzymes. Scand J Gastroenterol 2007;42:477-84.

54. Meropol NJ, Gold PJ, Diasio RB, Andria M, Dhami M, et al. Thymidine phosphorylase expression is associated with response to capecitabine plus irinotecan in patients with metastatic colorectal cancer. J Clin Oncol 2006;24:4051-3.

55. Koopman M, Vanderbosch S, Tinteren H, Ligtenberg MJ, Nagtegaal I, et al. Predictive and prognostic markers for the outcome of chemotherapy in advanced colorectal cancer, a retrospective analysis of the Phase III randomised CAIRO study. Eur J Cancer 2009;45:1999-2006.

56. Gustavsson B, Kaiser C, Carlsson G, Wettergren Y, Odin E, et al. Molecular determinants of efficacy for 5-FU-based treatments in advanced colorectal cancer: mRNA expression for 18 chemotherapy-related genes. Int J Cancer 2009;124:1220-6.

57. Ciaparrone M, Quirino M, Schinzari G, Zannoni G, Corsi DC, et al. Predictive role of thymidylate synthase, dihydropyrimidine dehydrogenase and thymidine phosphorylase expression in colorectal cancer patients receiving adjuvant 5-fluorouracil. Oncology 2006;70: 366-77.

58. Oi K, Makino M, Ozaki M, Takemoto H, Yamane N, et al. Immunohistochemical dihydropyrimidine dehydrogenase expression is a good prognostic indicator for patients with Dukes' C colorectal cancer. Anticancer Res 2004;24:273-9.

59. Lassmann S, Hennig M, Rosenberg R, Fontanini G, Vannozzi F, et al. Thymidine phosphorylase, dihydropyrimidine dehydrogenase and thymidylate synthase mRNA expression in primary colorectal tumors-correlation to tumor histopathology and clinical follow-up. Int $\mathrm{J}$ Colorectal Dis 2006;21:238-47.

60. Hotta T, Takifuji K, Taniguchi K, Sahara M, Yokoyama S, et al. The relationship between survival and the expression of dihydropyrimidine dehydrogenase in patients with colorectal cancer. Oncol Rep 2006;16:177-82.

61. Jensen SA, Vainer B, Sorensen JB. The prognostic significance of thymidylate synthase and dihydropyrimidine dehydrogenase in 
colorectal cancer of 303 patients adjuvantly treated with 5-fluorouracil. Int J Cancer 2007;120:694-701.

62. Soong R, Shah N, Salto-Tellez M, Tai BC, Soo RA, et al. Prognostic significance of thymidylate synthase, dihydropyrimidine dehydrogenase and thymidine phosphorylase protein expression in colorectal cancer patients treated with or without 5-fluorouracil-based chemotherapy. Ann Oncol 2008;19:915-9.

63. Kornmann M, Schwabe W, Sander S, Kron M, Sträter J, et al. Thymidylate synthase and dihydropyrimidine dehydrogenase mRNA expression levels: predictors for survival in colorectal cancer patients receiving adjuvant 5-fluorouracil. Clin Cancer Res 2003;9:4116-24.

64. Westra JL, Hollema H, Schaapveld M, Platteel I, Oien KA, et al. Predictive value of thymidylate synthase and dihydropyrimidine dehydrogenase protein expression on survival in adjuvantly treated stage III colon cancer patients. Ann Oncol 2005;16:1646-53.

65. Kornmann M, Link KH, Galuba I, Ott K, Schwabe W, et al. Association of time to recurrence with thymidylate synthase and dihydropyrimidine dehydrogenase mRNA expression in stage II and III colorectal cancer. J Gastrointest Surg 2002;6:331-7.

66. IKeguchi M, Makino M, Kaibara N. Thymidine phosphorylase and dihydropyrimidine dehydrogenase activity in colorectal carcinoma and patients prognosis. Langenbecks Arch Surg 2002;387:240-5.

67. Smorenburg CH, Peters GJ, Van Groeningen CJ, Noordhuis P, Smid K, et al. Phase II study of tailored chemotherapy for advanced colorectal cancer with either 5-fluouracil and leucovorin or oxaliplatin and irinotecan based on the expression of thymidylate synthase and dihydropyrimidine dehydrogenase. Ann Oncol 2006;17:35-42.

68. Toriumi F, Kubota T, Saikawa Y, Yoshida M, Otani Y, et al. Thymidylate synthetase (TS) genotype and TS/dihydropyrimidine dehydrogenase mRNA level as an indicator in determining chemosensitivity to 5-fluorouracil in advanced gastric carcinoma. Anticancer Res 2004;24:2455-63.

69. Terashima M, Irinoda T, Fujiwara H, Nakaya T, Takagane A, et al. Roles of thymidylate synthase and dihydropyrimidine dehydrogenase in tumor progression and sensitivity to 5-fluorouracil in human gastric cancer. Anticancer Res 2002;22:761-8.

70. Park JS, YoungYoon S, Kim JM, Yeom YI, Kim YS, et al. Identification of novel genes associated with the response to 5-FU treatment in gastric cancer cell lines using a cDNA microarray. Cancer Lett 2004;214:19-33.

71. Ishikawa Y, Kubota T, Otani Y, Watanabe M, Teramoto T, et al. Dihydropyrimidine dehydrogenase and messenger RNA levels in gastric cancer: possible predictor for sensitivity to 5-fluorouracil. Jpn J Cancer Res 2000;91:105-112.

72. Kodera Y, Ito S, Fujiwara M, Mochizuki Y, Nakayama G, et al. Gene expression of 5- fluorouracil metabolic enzymes in primary gastric cancer: correlation with drug sensitivity against 5- fluorouracil. Cancer Lett 2007;252:307-13.

73. Nishina T, Hyodo I, Miyaike J, Inaba T, Suzuki S, et al. The ratio of thymidine phosphorylase to dihydropyrimidine dehydrogenase in tumour tissues of patients with metastatic gastric cancer is predictive of the clinical response to 5'-deoxy-5-fluorouridine. Eur J Cancer 2004;40:1566-71.

74. Koizumi W, Okayasu I, Hyodo I, Sakamoto J, Kojima H. Clinical Study Group of Capecitabine. Prediction of the effect of capecitabine in gastric cancer by immunohistochemical staining of thymidine phosphorylase and dihydropyrimidine dehydrogenase. Anticancer Drugs 2008;19:819-24.

75. Shimizu T, Yamada Y, Yasui H, Shirao K, Fukuoka M. Clinical application of immunoreactivity of dihydropyrimidine dehydrogenase (DPD) in gastric scirrhous carcinoma treated with S-1, a new DPD inhibitory fluoropyrimidine. Anticancer Res 2005;25:2997-3001.

76. Ichikawa W, Takahashi T, Suto K, Shirota Y, Nihei Z, et al. Simple combinations of 5-FU pathway genes predict the outcome of metastatic gastric cancer patients treated by S-1. Int J Cancer 2006;119:1927-33.

77. Kim JY, Shin E, Kim JW, Lee HS, Lee DW, et al. Impact of intratumoral expression levels of fluoropyrimidine-Metabolizing enzymes on treatment outcomes of adjuvant S-1 therapy in gastric cancer. PloS One 2015;10:e0120324.

78. Sasako M, Terashima M, Ichikawa W, Ochiai A, Kitada K, et al. Impact of the expression of thymidylate synthase and dihydropyrimidine dehydrogenase genes on survival instage II/III gastric cancer. Gastric Cancer 2015;18:538-48.

79. Grau JJ, Caballero M, Monzò M, Muñoz-García C, Domingo-Domenech J, et al. Dihydropyrimidine dehydrogenases and cytidinedeaminase gene polymorphisms as outcome predictors in resected gastric cancer patients treated with fluoropyrimidine adjuvant chemotherapy. J Surg Oncol 2008;98:130-4.

80. Matsubara J, Nishina T, Yamada Y, Moriwaki T, Shimoda T, et al. Impacts of excision repair cross-complementing gene 1 (ERCC1), dihydropyrimidine dehydrogenase, and epidermal growth factor receptor on the outcomes of patients with advanced gastric cancer. Brit $\mathrm{J}$ Cancer 2008;98:832-9.

81. Shen XM, Zhou C, Lian L, Li LQ, Li W, et al. Relationship between the DPD and TS mRNA expression and the response to S-1-based chemotherapy and prognosis in patients with advanced gastric cancer. Cell Biochem Biophys 2015;71:1653-61.

82. Koizumi W, Tanabe S, Azuma M, Ishido K, Nishimura K, et al. Impacts of fluorouracil-metabolizing enzymes on the outcomes of patients treated with S-1 alone or S-1 plus cisplatin for first-line treatment of advanced gastric cancer. Int J Cancer 2010;126:162-70.

83. Tahara M, Ochiai A, Fujimoto J, Boku N, Yasui W, et al. Expression of thymidylate synthase, thymidine phosphorylase, dihydropyrimidine dehydrogenase, E2F-1, Bak, Bcl-X, and Bcl-2, and clinical outcomes for gastric cancer patients treated with bolus 5fluorouracil. Oncology Rep 2004;11:9-15.

84. Zhang C, Liu H, Ma B, Song Y, Gao P, et al. The Impact of the Expression Level of Intratumoral Dihydropyrimidine Dehydrogenase on Chemotherapy Sensitivity and Survival of Patients in Gastric Cancer: A Meta-Analysis. Dis Markers 2017;3:1-11.

85. Kondo N, Murakami Y, Uemura K, Sudo T, Hashimoto Y, et al. Prognostic impact of dihydropyrimidine dehydrogenase expression on pancreatic adenocarcinoma patients treated with S-1-based adjuvant chemotherapy after surgical resection. J Surg Oncol 2011;104:146-54.

86. Elander NO, Aughton K, Ghaneh P, Neoptolemos JP, Palmer DH, et al. Expression of dihydropirimidine dehydrogenase (DPD) and hENT1 predicts survival in pancreatic cancer. Brit J Cancer 2018;118:947-54.

87. Nakahara O, Takamori H, Tanaka H, Sakamoto Y, Ikuta Y, et al. Clinical significance of dihydropyrimidine dehydrogenase and 
thymidylate synthase expression in patients with pancreatic cancer. Int J Clin Oncol 2010;15:39-45.

88. Murakawa M, Aoyama T, Miyagi Y, Atsumi Y, Kazama K, et al. Clinical implications of dihydropyrimidine dehydrogenase expression in patients with pancreatic cancer who undergo curative resection with S-1 adjuvant chemotherapy. Oncol Lett 2017;14:1505-11.

89. Miyake K, Imura S, Yoshizumi T, Ikemoto T, Morine Y, et al. Role of thymidine phosphorylase and orotate phosphoribosyltransferase mRNA expression and its ratio to dihydropyrimidine dehydrogenase in the prognosis and clinico pathological features of patients with pancreatic cancer. Int J Clin Oncol 2007;12:111-9.

90. Saif MW, Hashmi S, Bell D, Diaso RB. Prognostication of pancreatic adenocarcinoma by expression of thymidine phosphorylase/ dihydropyrimidine dehydrogenase ratio and its correlation with survival. Expert Opin Drug Saf 2009;8:507-514.

91. Etienne MC, Chéradame S, Fischel JL, Formento P, Dassonville O, et al. Response to fluorouracil therapy in cancer patients: The role of tumoral dihydropyrimidine dehydrogenase activity. J Clin Oncol 1995;13:1663-70.

92. Tsuzuki M, Satomi T, Chiba H. Clinical significance of exression of thymidylate synthase and dihydropyrimidine dehydrogenase in oral squamous cell carcinoma. Oral Med Path 2010;14:135-41.

93. Hasegawa Y, Goto M, Hanai N, Ozawa T, Hirakawa H. Predictive biomarkers for combined chemotherapy with 5-fluorouracil and cisplatin in oro- and hipopharyngeal cancers. Mol Clin Oncol 2018;8:378-86.

94. Yu Z, Yang Q, Sun J, Zhen J. Dihydropirimidine dehydrogenase activity correlates with fluorouracil sensitivity in breast cancer. Exp Oncol 2007;29:192-6.

95. Horiguchi J, Takei H, Koibuchi Y, K Iijima, Ninomiya J, et al. Prognostic significance of dihydropyrimidine dehydrogenase expression in breast cancer. Br J Cancer 2002;86:222-5.

96. Hakamada Y, Tsuchida A, Arima M, Kubouchi T, Tokita H, et al. Prognostic predictors in breast cancer patients with postoperative 5-fluorouracil-based chemotherapy. Int J Mol Med 2005;16:309-14.

97. Tsunoda Y, Suzuki K, Tsunoda A, Takimoto M, Kusano M. Evaluation of 5-fluorouracil related genes in breast cancer to predict the effect of adjuvant therapy with oral fluorouracil derivatives. Oncol Rep 2010;23:771-7.

98. Zhao H, Huang H, Hu Z, Huang Y, Lin SX, et al. Evaluations of biomarkers associated with sensitivity to 5-fluorouracil and taxanes for recurrent/advanced breast cancer patients treated with capecitabine-based first-line chemotherapy. Anticancer Drugs 2012;23:534-42.

99. Qin F, Zhang H, Huang Y, Yang L, Yu F, et al. Effect of dihydropyrimidine dehydrogenase single nucleotide polymorphisms on prognosis of breast cancer patients with chemotherapy. Oncotarget 2017;8:112060-75.

100. Huang CL, Yokomise H, Kobayashi S, Fukushima M, Hitomi S, et al. Intratumoral expression of thymidylate synthase and dihydropyrimidine dehydrogenase in non-small cell lung cancer patients treated with 5-FU-based chemotherapy. Int J Oncol 2000; $17: 47-54$.

101. Okuda K, Tatematsu T, Yano M, Nakamae K, Yamada T, et al. The relationship between the expression of thymidylate synthase, dihydropyrimidine dehydrogenase, orotate phosphoribosyltransferase, excision repair cross-complementation group 1 and class III $\beta$-tubulin, and the therapeutic effect of S-1 or carboplatin plus paclitaxel in non-small-cell lung cancer. Mol Clin Onc 2018;9:21-9.

102. Clinical Pharmacogenetics Implemetation Consortium. Available from https://cpicpgx.org/ [Last accessed on 18 Jun 2019]

103. Lunenburg CA, Van Staveren MC, GelderblomH, Guchelaar HJ, Swen JJ. Evaluation of clinical implementation of prospective DPYD genotyping in 5-fluorouracil- or capecitabine-treated patients. Pharmacogenomics 2016;17:721-9.

104. Opdam FL, Swen JJ, Wessels JA, Gelderblom H. SNPs and haplotypes inDPYD and outcome of capecitabine-letter. Clin Cancer Res 2011;17:5837.

105. Rosmarin D, Palles C, Church D, Domingo E, Jones A, et al. Genetic markers of toxicity from capecitabine and other fluorouracil-based regimens: investigation in the QUASAR2 study, systematic review, and meta-analysis. J Clin Oncol 2014;32:1031-9.

106. Murphy C, Byrne S, Ahmed G, Kenny A, Gallagher J, et al. Cost Implications of Reactive Versus Prospective Testing for Dihydropyrimidine Dehydrogenase Deficiency in Patients With Colorectal Cancer: A Single-Institution Experience. Dose Response 2018;16:1-6.

107. Traorè S, Boisdron-Celle M, Hunault G, André T, Morel A, et al. DPD deficiency: medicoeconomic evaluation of pretreatment screening of 5-FU toxicity. J Clin Oncol 2012;30:410.

108. Scartozzi M, Maccaroni E, Giampieri R, Pistelli M, Bittoni A, et al. 5-Fluorouracil pharmacogenomics: still rocking after all these years? Pharmacogenomics 2011;12:251-65. 